\title{
Study Subject Magnetic Resonance Imaging
}

National Cancer Institute

\section{Source}

National Cancer Institute. Study Subject Magnetic Resonance Imaging. NCI Thesaurus.

Code C115539.

Magnetic resonance imaging of a study participant as part of a clinical trial. 